Neuroepidemiology 2012;38:217-218

DOI: $10.1159 / 000337162$

\section{Therapeutic Armamentarium and Health System Coverage of Multiple Sclerosis in Latin America}

Fernando Gracia ${ }^{\mathrm{a}}$, Blas Armien ${ }^{\mathrm{b}}$

a Neurology Section, Hospital Santo Tomás, and ${ }^{\mathrm{b}}$ Gorgas

Memorial Institute for Health Studies, Panama City, Panama

Epidemiological studies on multiple sclerosis in Latin America have been increasing over the last decade, promoted and encouraged by the Latin American Committee for Treatment and Research in Multiple Sclerosis (LACTRIMS) and societies or academic groups in each particular country. Although they present some methodological differences, recent publications and personal communications begin to provide a reasonable estimate of its frequency as well as some of its features.

Prevalence seems to range between 0.75 and 30 per 100,000 inhabitants. The incidence is between 0.6 and 2.2 per 100,000 inhabitants, which in general terms can be regarded as a lowprevalence disease [1-6]. There is scarce information on treatment availability, coverage and therapeutic effectiveness, treatment guides, the existence of academic, patients' and family associations, participation in clinical trials and national and international collaborative research projects or the disease burden. During a regional meeting on multiple sclerosis held in Cartagena, Colombia on May 20-21, 2011, and the Latin American Workshop on the Consensus for the Management of Multiple Sclerosis held in Quito, Ecuador on July 8-9, 2011, a voluntary survey was applied using a structural questionnaire (treatment availability and coverage; guides; academic, patients' and family associations; clinical trials and research projects) to some participating neurologists involved in multiple sclerosis and LACTRIMS delegates, considered opinion leaders in multiple sclerosis in their respective countries in order to obtain the information. Twenty countries were included (table 1). This data, although unofficial, gives an idea of the general situation of multiple sclerosis in Latin America. Analyses of the absolute and relative frequencies (percentages) were obtained. We concluded that significant advances have been made in the availability of drugs for treating acute crisis and the use of immunomodulators for relapsing/remitting multiple sclerosis; however, the population's access to these treatments is below 35\% in half of the countries. Among the countries that have the newest drugs available, only 4 have oral treatment with fingolimod and 11 with natalizumab. However, in more than $90 \%$ of the countries azathioprine, cyclophosphamide and mitoxantrone are available. On the other hand, all countries have founded associations that of-
Table 1. Therapeutic armamentarium and treatment of multiple sclerosis (MS) in 20 Latin American countries, 2011

\begin{tabular}{lc}
\hline Availability & $\%(\mathrm{n})$ \\
\hline Acute crisis & \\
Methylprednisolone & $100(20)$ \\
i.v. gamma globulin & $100(20)$ \\
Plasmapheresis & $90(18)$ \\
Immunomodulators & \\
Interferons & $100(20)$ \\
Glatiramer & $35(7)$ \\
Biosimilars & $35(7)$ \\
Natalizumab & $55(11)$ \\
Immunosuppressive drugs & \\
Azathioprine & $100(20)$ \\
Cyclophosphamide & $100(20)$ \\
Mitoxantrone & $90(18)$ \\
Fingolimod & $20(4)$ \\
MS patient coverage by health systems ${ }^{\mathrm{b}}$ & \\
Total & $100(9)$ \\
Medium-high & $65-80(2)$ \\
Low & $10-35(9)$ \\
Participation in associations/studies/guides & \\
Patient-family associations & $100(20)$ \\
MS centers/clinics & $60(12)$ \\
National clinical studies & $55(11)$ \\
International clinical studies & $60(12)$ \\
MS academic associations & $50(10)$ \\
Clinical trials & $50(10)$ \\
Treatment guides & $50(10)$ \\
Therapeutic effectiveness trials & $10(2)$ \\
\hline & \\
\hline
\end{tabular}

a North: Mexico; Caribbean: Cuba, Puerto Rico and Dominican Republic; Central America: Costa Rica, El Salvador, Guatemala, Honduras, Nicaragua and Panama; South America: Argentina, Bolivia, Brazil, Colombia, Chile, Ecuador, Paraguay, Peru, Uruguay and Venezuela.

${ }^{b}$ Ministry of Health, social security institutions and the private sector.

fer significant support to the different groups of patients and their families and $60 \%$ have a multiple sclerosis center/clinic. Only half of the countries have their own formal guidelines to unify the therapeutic approach from a methodological standpoint. There are few collaborative studies, whether national or

\section{KARGER}

Fax +4161306 1234 E-Mail karger@karger.ch www.karger.com
C 2012 S. Karger AG, Basel

0251-5350/12/0384-0217\$38.00/0

Accessible online at:

www.karger.com/ned
Dr. Fernando Gracia

Neurology Section

Hospital Santo Tomás

Panama City 0819-03752 (Panama)

Tel. +507 6613 6136, E-Mail fegra@ medicospaitilla.com 
international, as well as participation in clinical trials. This situation reflects how important it is to show public health authorities and decision-makers, that although multiple sclerosis has a low prevalence in our region, it has a major impact on society, the economy, employment and caregivers. It is important to ensure the accessibility of drugs and social benefits for these patients so that we can modify the natural course of the disease and significantly reduce the disease burden. It is urgent to carry out collaborative studies in Latin America with comparable methodologies in order to define epidemiological, clinical, health care and therapeutic aspects and promote research in neuroimmunology and genetics. Defining the disease burden is also a challenge.

\section{References}

1 Abad P, Perez M, Castro E, Alarcon T, Santibanez R, Diaz F: Prevalence of multiple sclerosis in Ecuador. Neurologia 2010;25:309-313.

-2 Corona T, Román GC: Multiple sclerosis in Latin America. Neuroepidemiology 2006;26:1-3.

-3 Gracia F, Castillo LC, Benzadon A, Larreategui M, Villareal F, Triana E, Arango AC, Lee D, Pascale JM, Gomez E, Armien B: Prevalence and incidence of multiple sclerosis in Panama (2000-2005). Neuroepidemiology 2009;32:287-293.

4 LACTRIMS: Sixth Latin American Congress on Multiple Sclerosis: Epidemiology in Latin America. LACTRIMS, Santiago, August 2010.

5 Rivera VM: Perspectives on multiple sclerosis in Latin America: a review. On behalf of the LACTRIMS steering committee (2005). CMSC Meet, Washington, May 2005.

6 Rivera VM: Multiple sclerosis in Latin America: reality and challenge. Neuroepidemiology 2009;32:294-295. 